\title{
ETNOBOTÁNICA DE CITRUS RETICULATA (RUTACEAE, AURANTIOIDEAE) NATURALIZADA EN LA ARGENTINA
}

\author{
PABLO C. STAMPELLA ${ }^{1}$, GUSTAVO DELUCCHI², HÉCTOR A. KELLER ${ }^{3} \&$ JULIO A. HURRELL ${ }^{4}$
}

\begin{abstract}
Summary: Stampella, P. C., G. Delucchi, H. A. Keller \& J. A. Hurrell. 2014. Ethnobotany of Citrus reticulata (Rutaceae, Aurantioideae) naturalized in Argentina. Bonplandia 23(2): 151-162.

In this contribution Citrus reticulata Blanco is confirmed as naturalized for Argentine Flora. Its updated synonymy, description, distribution, ecological data, cultivars and a key for their identification, and results of ethnobotanical studies aimed at understanding the naturalization process are included. This paper also contributes to the resolution of the current state of adventitious species of Citrus in Argentina.
\end{abstract}

Key words: Ethnobotany, naturalization, Citrus reticulata, Rutaceae, Argentina.

Resumen: Stampella, P. C., G. Delucchi, H. A. Keller \& J. A. Hurrell. 2014. Etnobotánica de Citrus reticulata (Rutaceae, Aurantioideae) naturalizada en la Argentina. Bonplandia 23(2): 151162.

En esta contribución se confirma como naturalizada para la Flora Argentina a Citrus reticulata Blanco. Se incluye su sinonimia actualizada, descripción, distribución y datos ecológicos, cultivares identificados y una clave para su identificación, y resultados de estudios etnobotánicos orientados hacia la comprensión del proceso de su naturalización. Se aporta, asimismo, a la resolución del estado actual de las especies adventicias de Citrus en la Argentina.

Palabras clave: Etnobotánica, naturalización, Citrus reticulata, Rutaceae, Argentina.

\section{Introducción}

El género Citrus L. (Rutaceae, Aurantioideae) comprende algunas de las especies de árboles frutales de mayor relevancia económica mundial. Es nativo de las zonas cálidas del sur y sudeste de Asia hasta las zonas templadas de China, Australia e islas del
Pacífico sudoccidental. Tiene una larga historia de domesticación, y especies ampliamente difundidas en cultivo, muchas veces, se han naturalizado en las zonas cálidas y templadocálidas del mundo (Scora, 1975; Roose et al., 1995; Spiegel-Roy \& Goldschmidt, 1996; Mabberley, 1998, 2001; Janick, 2005; Zhang \& Mabberley, 2008; Randall, 2012).

${ }^{1}$ Laboratorio de Etnobotánica y Botánica Aplicada (LEBA). Facultad de Ciencias Naturales y Museo, Universidad Nacional de La Plata, Calle 64 nro. 3, 1900-La Plata, Argentina. Becario UNLP. E-mail: pstampella@yahoo.com

${ }^{2}$ Facultad de Ciencias Naturales y Museo, Universidad Nacional de La Plata, Paseo del Bosque s.nro., 1900-La Plata, Argentina. E-mail: delucchi@fcnym.unlp.edu.ar

${ }^{3}$ Facultad de Ciencias Forestales, Universidad Nacional de Misiones - Instituto de Botánica del Nordeste, CC 209, 3400-Corrientes, Argentina. Investigador CONICET. E-mail: hakeller2000@yahoo.com.ar

${ }^{4}$ Laboratorio de Etnobotánica y Botánica Aplicada (LEBA). Facultad de Ciencias Naturales y Museo, Universidad Nacional de La Plata, Calle 64 nro. 3, 1900-La Plata, Argentina. Investigador CONICET. E-mail: juliohurrell@gmail. com 
La clasificación intragenérica de Citrus es compleja, debido a la presencia frecuente de procesos de hibridación y de apomixis (poliembrionía nucelar) (Xiang \& Roose, 1988; García et al., 1999). Dado que los híbridos pueden fijarse en distintos cultivares, el género presenta características morfológicas variables, situación que se refleja en las controversias sobre el número de especies: entre 61 y 162, según los autores (Swingle, 1943; Webber, 1943; Swingle \& Reece, 1967; Hodgson, 1965; Tanaka, 1954, 1966, 1969, 1977). Distintos estudios morfológicos, genéticos, filogenéticos y biomoleculares (Luro et al., 1995; Nicolosi et al., 2000; Nicolosi, 2007; Bayer et al., 2009; Kyndt et al., 2010; Marlykynti et al., 2011; Uzun \& Yesiloglu, 2012, Kumar et al., 2013; Penjor et al., 2013, entre otros) aportaron a la comprensión del género, y en la actualidad se aceptan entre 20 y 25 especies (Mabberley, 1997, 1998, 2001, 2004), criterio adoptado en la presente contribución.

Para la Argentina, se han citado cuatro taxones naturalizados: 1. C. $\times$ aurantium L. Grupo 'Naranja amarga' [C. maxima (Burm.) Merr. $\times$ C. reticulata Blanco], en Jujuy, Salta, Tucumán, Formosa, Chaco, Misiones, Corrientes, Santa Fe, Córdoba y Buenos Aires (Miranda et al., 2000; Bacigalupo, 2005; Seo \& Xifreda, 2008; IBODA, 2014); 2. C. × aurantium L. Grupo 'Pomelo' (=C. $\times$ paradisi Macfad.) [(C. maxima $\times C$. reticulata $) \times C$. maxima $]$, en la provincia de Buenos Aires (Hurrell et al., 2010); 3. C. × taitensis Risso [C. reticulata $\times$ C. medica L., o bien $C$. reticulata $\times C$. $\times$ limon (L.) Osbeck], en Misiones (Stampella et al., 2013); 4. C. trifoliata L. [= Poncirus trifoliata (L.) Raf.], en Entre Ríos y Buenos Aires (Bacigalupo, 2005; Seo \& Xifreda, 2008; IBODA, 2014). Asimismo, el "apepú" (Citrus $\times$ aurantium L. Grupo 'Naranja amarga') es citada como naturalizada en Paraguay (Gade, 1976).

En esta contribución se confirma la presencia de Citrus reticulata Blanco, "mandarino", como naturalizada en nuestro país. Esta especie fue mencionada como posible naturalizada en el noroeste de Corrientes por Pedersen (1995), pero sin material de referencia que acredite dicha afirmación. C. reticulata, considerada en su sentido amplio (Mabberley, 2004; Bayer et al., 2009; Uzun \& Yesiloglu, 2012), incluye numerosos cultivares, algunos de los cuales se han identificado como especies distintas, según diferentes criterios de demarcación (Fang et al., 1998; Moreira Novelli et al., 2000; Baig et al., 2009; Kujawska et al., 2012). Asimismo, este trabajo aporta a la resolución del estado actual del conocimiento sobre las especies adventicias de Citrus en la Argentina, y a los aspectos etnobotánicos referidos en particular a la naturalización de $C$. reticulata en el país.

\section{Materiales y Métodos}

Los resultados de esta contribución han sido obtenidos en diversos viajes de campo, realizados en el lapso 2007-2013, abarcando diferentes períodos del ciclo anual, con fines tanto florísticos como etnobotánicos, en el Noreste argentino y en la región rioplatense. Los materiales de referencia que documentan el trabajo realizado corresponden a ejemplares naturalizados y fueron depositados en los herbarios CTES y LP, y en las colecciones del LEBA (Laboratorio de Etnobotánica y Botánica Aplicada, Facultad de Ciencias Naturales y Museo, UNLP). Asimismo, se consultaron los herbarios BAA, LPAG y SI y bases de datos como la Flora del Cono Sur (IBODA, 2014) y la Lista de Espécies da Flora do Brasil (Pirani, 2014); además: New South Wales Flora on Line (2013), Plants Profile USDA (2013); The Plant List (2013) y Tropicos.org (2014). La sinonimia presentada se refiere sólo a nombres de uso frecuente en la Argentina y países vecinos. Para una sinonimia completa, ver Zhang \& Mabberley (2008).

En la provincia de Misiones se desarrollaron distintas metodologías etnobotánicas cualitativas habituales: observación participante, listados libres, entrevistas abiertas y semiestructuradas (Quinlan, 2005; Stepp, 2005; Albuquerque et al., 2010, 2014; Etkin \& Ticktin, 2010). Se entrevistaron informantes de las localidades donde se coleccionó material de referencia, 36 personas en total, relacionadas con ambientes rurales y periurbanos, 20 de sexo femenino y 16 de sexo masculino, entre 18 y 90 años de edad. Dichas entrevistas fueron grabadas y depositadas en el Laboratorio de Etnobotánica y Botánica Aplicada (LEBA). Dada la diversidad cultural propia de Misiones, los informantes se autodefinen, en 
su mayoría, como "criollos", descendientes de españoles, italianos, portugueses, paraguayos, entre otros. Es de destacar la presencia de descendientes de inmigrantes polacos, ucranianos y alemanes, llegados en las primeras décadas del siglo XX, y de guaraníes M'bya. Los resultados revelaron un amplio consenso de los informantes. Las temáticas abordadas en las entrevistas se orientaron al uso y manejo local de las plantas, información sobre su introducción, ambientes donde se considera que crece de forma espontánea, tiempo de permanencia y aspectos referidos a la dispersión de las semillas, con el fin de aportar a la comprensión del proceso de su naturalización. Esta estrategia metodológica, que implica una contribución efectiva de la etnobotánica al estudio de la naturalización de especies exóticas, ha sido desarrollada por los autores con anterioridad (Hurrell et al., 2012; Keller et al., 2013; Stampella et al., 2013; Hurrell \& Delucchi, 2014, entre otros).

\section{Resultados y Discusión}

Citrus reticulata Blanco, Fl. Filip.: 610, 1837.

C. $\times$ nobilis Lour., Fl. Cochinch. 2: 466, 1790; $C$. deliciosa Ten., Index Sem. Hort. Neapol. 9, 1840; $C$. $\times$ aurantium L. var. tachibana Makino, Bot. Mag. (Tokyo) 15: 167, 1901; C. depressa Hayata, Icon. Pl. Formosan. 8: 16, 1919; C. unshiu Marcov., Izv. Sukhumsk. Sadovoi Sel'skokhoz. Opyt. Stantsii 1921(2): 5, 1921; C. tachibana (Makino) Yu.Tanaka, Bult. Sci. Fak. Terk. Kjusu Imp. Univ. 2: 52, 1926; $C$. sunki Yu. Tanaka, Mem. Tanaka Citrus Exper. Stat. 1(1): 42, 1927; C. erythrosa Yu. Tanaka, Stud. Citrol. 3: 184, 1930; C. reshni Yu.Tanaka, Stud. Citrol. 8: 72, 1937; C. reticulata Blanco var. austera Swingle, J. Wash. Acad. Sci. 32: 25, 1942; C. × aurantium L. f. deliciosa (Ten.) M. Hiroe, Forest Pl. Hist. Jap. Is. 1: 226, 1974.

Iconografia: Besler, Hortus Eystettensis 2: tab. 143, fig. 1, 1640 (sub Poma aurantia).

Arbolitos perennifolios de 2-6 m alt.; ramas jóvenes flexibles, delgadas, angulosas, con pocas espinas axilares solitarias. Hojas alternas, 1-folioladas (hojas compuestas reducidas a un único folíolo); pecíolos articulados, marginados o angostamente alados; láminas elípticas u ovadas, 3-8 cm long. $\times 1,5-4 \mathrm{~cm}$ lat., ápice emarginado, borde crenulado o rara vez entero, base atenuada, coriáceas a subcoriáceas, con glándulas oleíferas pelúcidas, verde oscuras, brillantes. Flores axilares, solitarias o en fascículos 2-3-floros, fragantes. Cáliz cupuliforme, irregularmente 3-5-lobado, 3-4 mm long., subglabro. Pétalos 4-5, imbricados, gruesos, glandulosos, elípticos u oblongos, 0,8-1,5 cm long., blancos, a menudo rosados por fuera. Disco con glándulas nectaríferas. Estambres 20-25. Ovario con 5-14 lóculos pluriovulados; estilo cilíndrico, largo, delgado; estigma claviforme. Fruto hesperidio subgloboso, a menudo deprimido, 5-10 cm diám., anaranjado, rojizo o amarillento; pericarpo muy delgado a grueso, fácil de remover, liso o rugoso; segmentos 7-14, raro más, pulpa dulce a ácida, a veces amarga. Semillas ovoides, agudas en el ápice, lisas, $c a .1 \mathrm{~cm}$ long.; poliembrionía. $2 n=18,36$ (Tropicos.org, 2014).

Distribución geográfica: Especie posiblemente originaria del sudeste de China y sur de Japón y de la India, ampliamente difundida en cultivo. Comprende más de 200 cultivares (Cornélio et al., 2003; Mabberley, 2004; Nicolosi, 2007; Zhang \& Mabberley, 2008). Se halla naturalizada en Europa (España, Reino Unido), Mozambique, Sudeste asiático, Nueva Zelanda, Australia, Estados Unidos, Ecuador, Galápagos y Brasil (Laguna Lumbreras \& Mateo Sanz, 2001; Randall, 2012; Pirani, 2014).

En la Argentina, diversos cultivares de $C$. reticulata se cultivan en cuatro regiones: noroeste (Jujuy, Salta, Tucumán, Catamarca), norte (Formosa, Chaco), nordeste (Misiones, Corrientes, Entre Ríos) y el noreste de la provincia de Buenos Aires (SENASA, 2012). Esta especie se confirma aquí como naturalizada para el país, en la provincia de Misiones y en la Ciudad Autónoma de Buenos Aires. Referencias anteriores sobre el carácter naturalizado de esta especie en Corrientes (Pedersen, 1995) y Salta (SIB, 2014) no han sido confirmarlas, debido a la ausencia de materiales de herbario de referencia.

Fenología, polinización y dispersión en nuestro país: Florece en primavera-verano y fructifica en otoño-invierno. De acuerdo con las observaciones realizadas en el terreno, la 
polinización es entomófila, en especial melitófila; la dispersión del fruto es fundamentalmente zoocora, y quizás también hidrocora (las semillas son llevadas por las corrientes de agua). Los frutos son consumidos por aves (Psittacidae, Corvidae, Cotingidae, Thraupidae, entre otras familias), por pequeños mamíferos (Dasyprocta azarae: "agut'́," Cuniculus paca: "paca", especies de Dasypodidae: "tatú"), y por el ganado (vacuno, porcino, ovino). La antropocoria también es relevante: tanto niños como adultos comen la pulpa y desechan las semillas.

Importancia económica: Es una de las especies de Citrus de mayor relevancia económica a nivel mundial, y presenta el mayor rango de aclimatación de las especies cultivadas del género (Koehler-Santos et al., 2003). En China, se cultivo se remonta a 4000 años; no obstante, se introdujo en Occidente tardíamente en relación a otros cítricos, en 1805 (Ramón-Laca, 2003; Nicolosi, 2007). En América, los primeros registros podrían corresponder a Brasil, en la primera mitad del siglo XIX (Saint Hilaire, 1825; Martius, 1843). En la Argentina, su introducción data de fines del mismo siglo (Hieronymus, 1882; Burmeister, 1899; Hirschhorn, 1930; Patiño, 1963).

Se ha señalado como una de las especies del género más cultivada en el país, valorada como frutal y asimismo ornamental (Dimitri, 1988). La planta es melífera (Zamudio \& Hilgert, 2011; Kujawska et al., 2012). La pulpa comestible se consume fresca, en jugos y mermeladas; el epicarpo se usa como saborizante en gastronomía y licorería. La esencia se emplea en perfumería. En medicina popular se utiliza como remedio antiescorbútico, digestivo, antiespasmódico, depurativo, sedante, entre otros (Hurrell et al., 2010). Presenta actividad anticancerígena (Kim et al., 2005), antiinflamatoria (Ho \& Lin, 2008), antimicrobiana (Sultana et al., 2012) y antioxidante (Zhang et al., 2014).

Material examinado: ARGENTINA. Ciudad Autónoma de Buenos Aires: Reserva Ecológica Costanera Sur, 8-XII-2007, Hurrell et al. 6634 (LP); 2-V-2014, Delucchi et al. 3615 (LP). Misiones: Dep. Concepción de la Sierra, Ruinas de Santa María la Mayor, 15-VI-2010, Stampella \& Hilgert 5 (LEBA); 15-IX-2010, Stampella 43 (LP), 24IV-2012, Stampella 99 (LP); Ruinas de Santos Mártires, $27^{\circ} 48^{\prime} 11,8^{\prime}$ S 55 $5^{\circ} 24^{\prime} 55,4$ " W, 15-VI2010, Stampella 17 (LEBA); Paraje Cerro Mártires, ca. chacra Familia Bueno, 21-VII-2011, Stampella 83 (LEBA); Dep. Iguazú, Colonia Gobernador J. J. Lanusse, 17-III-2011, Kujawska 135 (CTES). Dep. Montecarlo, Colonia Guatambú, 9-V-2011, Keller 10354 (CTES); calle Ritter, entre ruta 12 y vivero del pionero Ritter, 7-VII-2013, Keller 11492 (CTES); Dep. San Ignacio, Teyú Cuaré, ca. chacra Don Colman, 29-III-2011, Stampella et al. 59 (LEBA); Ruinas de San Ignacio Miní, 11-IX-2010, Stampella 36 (LP).

Obs.: C. reticulata se diferencia de los otros taxones del género naturalizados en la Argentina a través de los siguientes caracteres.

\section{Clave para diferenciar los Citrus naturalizados en la Argentina}

1. Arbustos caducifolios. Hojas trifolioladas, pecíolos levemente alados. Flores solitarias. Frutos hasta de $5 \mathrm{~cm}$ diám., epicarpo pubescente, amarillo-anaranjado. Citrus trifoliata

1'. Árboles o arbolitos perennifolios. Hojas 1-folioladas, pecíolos leve a anchamente alados. Flores en racimos paucifloros. Frutos de más de $5 \mathrm{~cm}$ diám., epicarpo glandular, no pubescente, anaranjado o amarillo.

2. Árboles con láminas foliares ovadas, pecíolos anchamente alados. Frutos subglobosos, epicarpo y mesocarpo grueso, amargo.

3. Frutos hasta de $8 \mathrm{~cm}$ diám., epicarpo rugoso, anaranjado. Citrus $\times$ aurantium (Grupo 'Naranjo amargo')

3'. Frutos de más de $8 \mathrm{~cm}$ diám., epicarpo ligeramente rugoso, amarillo.

Citrus $\times$ aurantium (Grupo 'Pomelo')

2'. Arbolitos con láminas foliares oblanceoladas, pecíolos levemente alados. Frutos depreso-globosos, epicarpo y mesocarpo delgado, no amargo.

4. Follaje laxo. Pimpollos blancos con tintes purpúreos. Pulpa ácida a la madurez. .... Citrus $\times$ taitensis

4'. Follaje denso. Pimpollos blancos sin tintes purpúreos. Pulpa usualmente dulce a la madurez. Citrus reticulata 
Cultivares identificados: Entre las plantas coleccionadas fueron identificados dos cultivares distintos: el cv. 'Cleopatra' [Keller 10354 y 11492 (CTES)], y el cv. 'Común de Concordia' [el resto de los ejemplares].

C. reticulata cv. 'Cleopatra' (Fig. 1) se ha identificado a menudo en la literatura como $C$. reshni Hort. ex Tan. (Luro et al., 1995; Sánchez Damas et al., 2006; Baig et al., 2009; Hernández et al., 2010; Kujawska et al., 2012); no obstante, esta especie ha sido asimilada a $C$. reticulata (Samaan, 1982; Fang et al., 1998; Rivera Núñez et al., 1998; Cheng et al., 2005; Froelicher et al., 2011). Crece adventicio en España (Laguna Lumbreras \& Mateo Sanz, 2001). Fue introducido a la Argentina desde Brasil en la década de 1950, junto con otros pies de injerto, luego de la epidemia de virus de la tristeza de los cítricos, que arrasó los cultivos comerciales sobre pie de "naranjo amargo" (Banfi, 1954; Banfi \& Beñatena, 1954; Palacios, 1978), también se cultiva como ornamental.

C. reticulata cv. 'Común de Concordia'
(Figs. 1 y 2) ha sido con frecuencia tratada con el nombre de $C$. deliciosa Ten. (Tanaka, 1977; Machado et al., 1996; Andrés et al., 2001; Ramos-Hurtado et al., 2006; Dalla Rosa et al., 2012), en la actualidad, sinónimo de $C$. reticulata (Zhang \& Mabberley, 2008). Se introdujo en la Argentina a fines del siglo XIX, seguramente desde Brasil, donde recibió los nombres de "mandarino común" o "del Mediterráneo" (región donde presentó amplísima difusión), "naranjo tangerino" o "naranjo fino" (Hieronymus, 1882; Burmeister, 1899; Patiño, 1963). Su cultivo se inició en Concordia (Entre Ríos), luego se expandió a Corrientes y, en la década de 1930, se desarrolló en Misiones (Hirschhorn, 1930; Palacios, 1978). Fue la variedad más cultivada en nuestro país hasta principios de la década de 1980, cuando fue desplazada por otras, por ejemplo, el cv. 'Clementina' (Samaan, 1982; Anderson, 1996).

Los cultivares identificados en la presente contribución pueden distinguirse por medio de la siguiente clave.

\section{Clave para diferenciar los cultivares naturalizados en Misiones y la Ciudad Autónoma de Buenos Aires}

1. Follaje verde claro, hojas ovadas a oblanceoladas, agudas a acuminadas. Espinas hasta de $1 \mathrm{~cm}$ de longitud. Frutos generalmente pequeños, rojizos a la madurez (julio-agosto), pericarpo levemente adherente, mesocarpo hasta de $5 \mathrm{~mm}$ de grosor. ........................................... C. reticulata cv. 'Cleopatra'

1'. Follaje verde oscuro, hojas oblanceoladas, marcadamente acuminadas. Espinas mayores de $1 \mathrm{~cm}$ de longitud. Frutos medianos, amarillo-anaranjado a la madurez (abril-mayo), pericarpo suelto, mesocarpo hasta de $3 \mathrm{~mm}$ de grosor. C. reticulata cv. 'Común de Concordia'

Etnobotánica y naturalización: En Misiones, $C$. reticulata cv. 'Cleopatra' se denomina "mandarina colorada" (por su pericarpo maduro rojizo), "mandarina agosto" (mes de maduración de los frutos), "mandarina limón" (por su sabor ácido), y "mandarina de injerto" (por su uso como pie de injerto para el cultivo de otros cítricos). Los frutos se consumen bien maduros, porque se endulzan a último momento, luego de varias heladas. Los frutos inmaduros se consumen como limón, para sazonar comidas y el tereré. Se encuentra naturalizado en selvas secundarias o capueras (que difieren del monte sólo por el menor diámetro de tronco de los árboles). Muchos agricultores pequeños y medianos del centro de la provincia abandonaron sus plantaciones de cítricos, o las remplazaron luego de una intensa helada con nevisca, ocurrida el 17 de julio de 1975. Además de los pies de injerto originales, devenidos en ejemplares adultos, se observa una abundante regeneración natural, a distancias tales que su presencia evidencia su dispersión zoocora.

C. reticulata cv. 'Común de Concordia', recibe en Misiones los nombres locales de "mandarina común", "mandarina criolla", "mandarina casera", "mandarina silvestre" y "mandarina del monte". Según los pobladores locales, los frutos se consumen de forma directa y se emplean para elaborar dulce, con el agregado de "cáscaras" de C. × limon (L.) Osbeck, "limón amarillo", que suplementan la baja proporción de pectinas del mesocarpo 

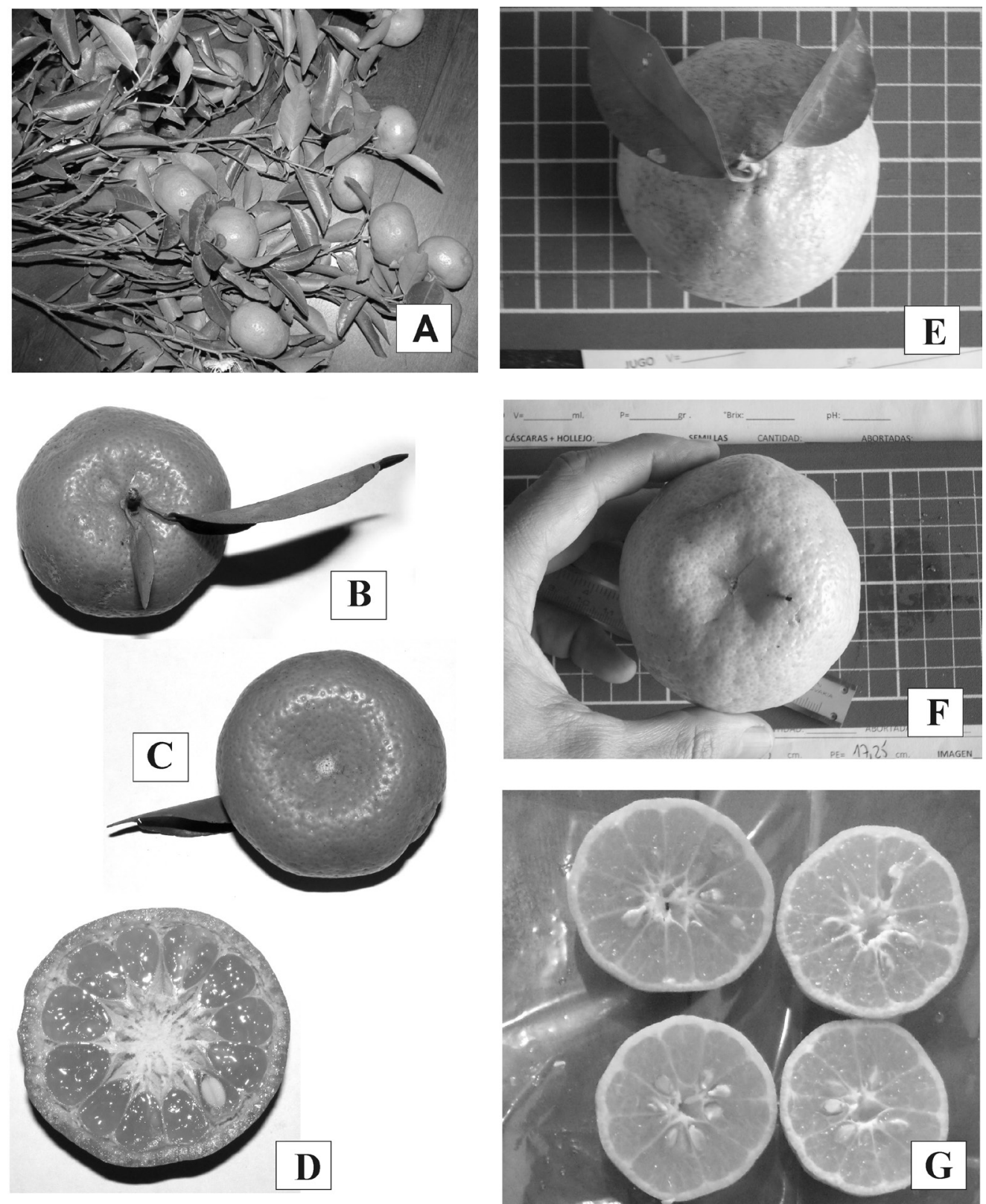

Fig. 1. Citrus reticulata cv. 'Cleopatra'. A: Rama con frutos. B: Fruto visto desde arriba. C: Fruto visto desde abajo. D: Corte transversal de fruto (Fotos: H. A. Keller). C. reticulata cv. 'Común de Concordia'. E: Fruto visto desde arriba. F: Fruto visto desde abajo. G: Corte transversal de frutos (Fotos: P. C. Stampella).

de las mandarinas. La infusión de hojas y pericarpos se utiliza como remedio febrífugo, antigripal, emético y sedante; el cocimiento, como anticatarral, antiséptico y analgésico para dolores de muelas y de cintura; el jugo se considera refrescante, sedante y digestivo.
Este cultivar se halla naturalizado dentro de los montes, en especial, en los montes bajos de las zonas de afloramientos rocosos con suelo de escaso desarrollo, caracterizadas por MartínezCrovetto (1963) como pertenecientes al Distrito del Urunday de la Selva Paranaense. Los 

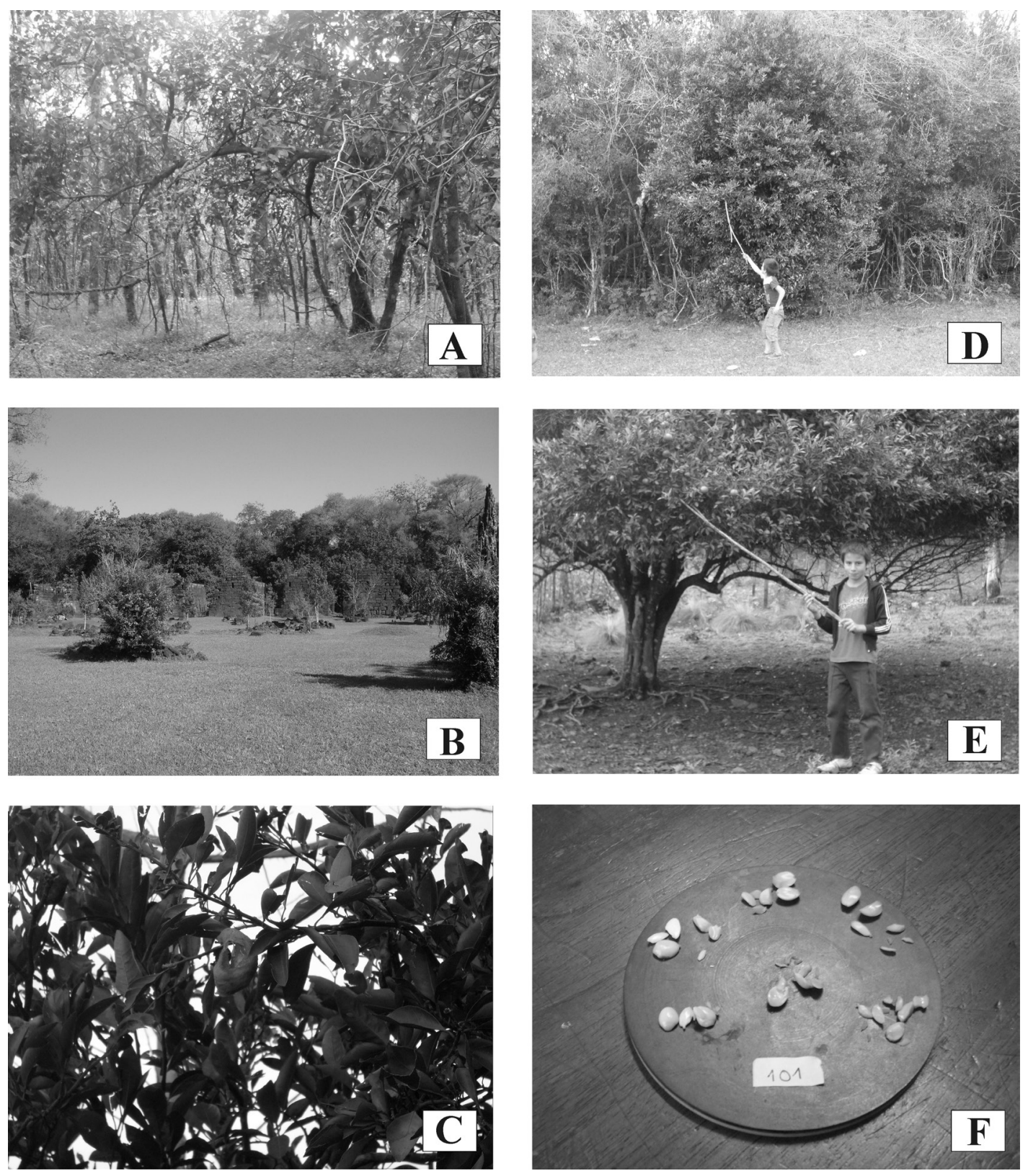

Fig. 2. Citrus reticulata cv. 'Común de Concordia'. A: Ejemplares naturalizados en el monte. B: Plantas en ruinas de Santa María la Mayor. C: Fruto comido por aves. D-E: "Ganchos” para extraer frutos. F: Semillas con embriones múltiples (Fotos: P. C. Stampella).

ejemplares de los bordes de monte (en contacto con los campos o potreros) presentan mayor cantidad de frutos, más grandes y dulces a la madurez que los de los ejemplares del interior del monte. Estos se consideran "viciosos" por su mayor desarrollo vegetativo y baja producción de frutos pequeños, sin jugo y sin sabor. Los pobladores emplean "ganchos", instrumentos improvisados de madera, para extraer los frutos del ramaje denso de estas plantas, y evitan asimismo sus espinas. La recolección se realiza en otoño, cuando maduran las mandarinas y son 
escasos otros frutos comestibles de especies silvestres, como Eugenia uniflora L., "pitanga", y Myrcianthes pungens (O. Berg) D. Legrand, "guabiyú". Las plantas de los bordes de monte también son elegidas como "semilleros", si se desea cultivar mandarinos en huertos familiares. Este cultivar crece, además, entre escombros de las ruinas de Santa María la Mayor, un caso atípico de naturalización de leñosas en ambientes no boscosos.

Es de destacar la presencia de plantas enfermas en la zona. Los pobladores se refieren a estas como "pesteadas" y aseguran que, como ocurre con otros cítricos, perduran pocos años, las ramas se secan y los brotes se deforman. Estos y otros síntomas concuerdan con la enfermedad bacteriana de los cítricos llamada Huanglongbing (HLB), de reciente aparición en Misiones (Cortese, 2011).

En la Ciudad Autónoma de Buenos Aires, $C$. reticulata ha sido hallada en bordes de caminos interiores de la Reserva Ecológica de la Costanera Sur. El origen in situ de los ejemplares no responde a la introducción en cultivo y posterior naturalización, como ocurre en Misiones. Su presencia podría deberse tanto a la diseminación ornitocora como a la antropocora: los visitantes ocasionales de la reserva suelen arrojar las semillas en los bordes de los caminos al consumir los frutos.

\section{Conclusiones}

La naturalización de especies exóticas, sobre todo, las que pueden devenir invasoras, es una problemática ampliamente difundida en diversos países del mundo, y se ha generado en la última década una terminología específica para definir distintas situaciones dentro del proceso de naturalización (Richardson et al., 2000, 2011; Pyšek et al., 2004; Pyšek \& Richardson, 2006; Randall, 2012; Hurrell \& Delucchi, 2014). En este marco, C. reticulata en la Argentina debe considerarse una especie naturalizada, en tanto se reproduce y conforma poblaciones autosustentables, con varios ciclos de vida, que se expanden por sus propios medios (por vía sexual o vegetativa), durante varias décadas, sin la intervención humana (en términos de actividades culturales).
Las poblaciones halladas en Misiones claramente resultan remanentes de antiguos cultivos, con rebrote de los pies de injertos (al morir los injertos), y una consecuente naturalización por la vía de mecanismos de dispersión zoocora (incluida la antropocoria), y posiblemente hidrocora. Dada la antigüedad de los cultivos originales en la provincia, las poblaciones naturalizadas son autosustentables, con una sucesión de varios ciclos de vida sin intervención humana. No se trata de una especie invasora, dado que su expansión no es agresiva ni comprometería la integridad de las comunidades de las floras locales. Los informantes confirman la situación de la especie, dado que siempre se ha considerado integrada al paisaje local, como sugieren los nombres vernáculos "mandarina del monte" y "mandarina silvestre" (por oposición a los mandarinos de las plantaciones). Los pobladores locales asimismo recolectan los frutos de estas plantas de igual modo que los de las especies arbóreas nativas.

Los ejemplares encontrados en la Ciudad Autónoma de Buenos Aires se consideran asimismo naturalizados, dado que perduran y completan sus ciclos vitales, al menos por dos décadas, sin la intervención humana. La situación es muy distinta a la de Misiones, porque la mayoría de las formaciones boscosas de la Reserva Costanera Sur están constituidas principalmente por especies arbóreas exóticas. Si bien se requieren más datos a lo largo del tiempo, así como una mayor comprensión de la composición y estructura florística de las formaciones boscosas locales aludidas, el concepto de especie naturalizada se ajusta al caso de $C$. reticulata.

\section{Agradecimientos}

Los autores agradecen a las comunidades de los parajes Cerro Mártires, Caá Guazú y La Corita (Santa María, Concepción de la Sierra), Teyú Cuaré, Puerto Viejo y pueblo de San Ignacio (San Ignacio); al personal de la Escuela No 590 "Víctor Mercante" del paraje Cerro Mártires y de las ruinas de Santa María la Mayor (Concepción de la Sierra); y a todos los informantes entrevistados que gentilmente 
compartieron sus conocimientos. A Fernando, Alejandro, Matías, Agustín, Julián, Ángel, Kevin, Diosnel, Elías y Claudio, por la ayuda brindada en los trabajos de campo y en el relevamiento de datos. A Norma Hilgert, $M$. Lelia Pochettino, Violeta Furlán, Marcos Núñez, Rodrigo Dutra y Pablo Cabanillas por su apoyo logístico y colaboración en los viajes de estudio; al último de ellos, además, por su ayuda en la elaboración de las láminas. A los dos revisores anónimos por sus acertadas sugerencias que enriquecieron este trabajo.

\section{Bibliografía}

ALBUQUERQUE, U. P., L. R. FAVIAS PIAVA \& L. V. FERNANDEZ (eds.). 2010. Métodos e técnicas na pesquisa etnobiológica e etnoecológica. NUPPEA, Recife. 559 pp.

ALBUQUERQUE, U. P., L. V. F. CRUZ DA CUNHA, R. F. PAIVA DE LUCEA \& R. N. ALVES (eds.). 2014. Methods and Techniques in Ethnobiology and Ethnoecology. Springer, New York. 480 pp.

ANDERSON, C. 1996. Variedades cultivadas en el área del Río Uruguay. En Fabiani, A., R. Mika, L. Larocca y C. Anderson (eds.), Manual para productores de naranja y mandarina de la región del Río Uruguay, pp. 63-92. Diversificación Productiva, Manual Serie A Nro. 2. INTA, Concordia.

ANDRÉS, C., B. LUQUE \& M. J. DÍEZ. 2001. Morfología polínica de las especies de cítricos cultivadas en Andalucía Occidental (España). Acta Bot. Malacitana 26: 69-77.

BACIGALUPO, N. M. 2005. Rutaceae. En Burkart, A. \& N. M. Bacigalupo (eds.), Fl. Ilustr. Entre Ríos. Colecc. Ci. Inst. Nac. Tecnol. Agropecu. 6(4b): 56-64.

BAIG, M., S. GREWAL \& S. DHILLON. 2009. Molecular characterization and genetic diversity analysis of citrus cultivars by RAPD markers. Turk. J. Agric. For. 33: 375-384.

BANFI, A. 1954. Difusión de buenas variedades cítricas. IDIA 90-92: 102.

— \& H. BEÑATENA. 1954. Nuevas variedades en la colección de Citrus. IDIA 90-92: 101-102.

BAYER, R., D. J. MABBERLEY, C. MORTON, C. MILLER, I. K. SHARMA, B. E. PFEIL, S. RICH, R. HITCHCOCK \& S. SYKES. 2009. A molecular phylogeny of the orange subfamily (Rutaceae: Aurantioideae) using nine cpDNA sequences. Amer. J. Bot. 96: 668-685.

BURMEISTER, C. 1899. Memoria sobre el territorio de Misiones. Ministerio de Agricultura de la República Argentina. Peuser, Buenos Aires. 77 pp.
CHENG, Y., M. C. VICENTE, H. MENG, W. GUO, N. TAO \& X. DENG. 2005. A set of primers for analyzing chloroplast DNA diversity in Citrus and related genera. Tree Physiology 25: 661-672.

CORNÉliO, M., A. FIGUEIRÔA, K. SANTOS, R. CARVALHO, W. SOARES FILOHO \& $\mathrm{M}$. GUERRA. 2003. Chromosomal relationships among cultivars of Citrus reticulata Blanco, its hybrids and related species. Plant Syst. Evol. 240: 149-161.

CORTESE, P. 2011. Situación actual de las acciones de vigilancia y prevención para el Huanglongbing en la Argentina. SENASA, Buenos Aires. 23 pp.

DALlA ROSA, R., G. A. NAVA, A. L. PIVA, E. J. MEZZALIRA \& D. PAULUS. 2012. Poda e raleio manual de tangerineira (Citrus deliciosa Ten.) cv. Montenegrina no Sudoeste do Paraná. Revista Ceres 59: 254-261.

DIMITRI, M. J. 1988. Rutáceas. En Dimitri, M. J. (ed.), Encicl. Argent. Agric. Jard. (3ra. ed. 3) 1(2): 667676. Acme, Buenos Aires.

ETKIN, N. L. \& TICKTIN, T. 2010. Advancing an ethno-ecological perspective that integrates theory and method in ethnobotany. En Albuquerque, U.P. $\&$ N. Hanazaki (eds), Recent developments and case studies in Ethnobotany, pp. 33-57. SBEE/NUPEEA, Recife.

FANG, D., R. KRUEGER \& M. ROOSE. 1998. Phylogenetic relationships among selected Citrus germplams accessions revealed by inter-simple sequence repeat (ISSR) markers. J. Amer. Soc. Hort. Sci. 123: 612-617.

FROELICHER, Y., M. WAFA, J. B. BASSENE, G. COSTANTINO, M. KAMIRI, F. LURO, R MORILLON \& P. OLLITRAULT. 2011. New universal mitochondrial PCR markers reveal new information on maternal citrus phylogeny. Tree Genet. Genomes 7: 49-61.

GADE, D. W. 1976. Naturalization of plant aliens: the volunteer orange in Paraguay. J. Biogeography 3: 269-279.

GARCÍA, R., M. J. ASÍNS, J. FORMER \& E. A. CARBONELL. 1999. Genetic analysis of apomixes in Citrus and Poncirus by molecular markers. Theor. Appl. Genet. 99: 511-518.

HERNÁNDEZ, R. M., E. DIOSDADO, J. C. CABRERA \& F. COLL. 2010. Efecto de los biorreguladores del crecimiento en la embriogénesis somática de mandarina Cleopatra (Citrus reshni Hort. ex Tan.). Cultivos Tropicales 31: 32-38.

HIERONYMUS, J. 1882. Plantae Diaphoricae Florae Argentinae. Kraft, Buenos Aires. 404 pp.

- 1930. Contribución al estudio de los Citrus en la provincia de Corrientes. Revista Fac. Agron. La Plata 19: 71-99.

HO, S. C. \& C. C. LIN. 2008. Investigation of heat treating conditions for enhancing the antiinflammatory activity of citrus fruit (Citrus 
reticulata) peels. J. Agric. Food Chem. 56: 79767982.

HODGSON, R. W. 1965. Taxonomy and nomenclature in the Citrus fruits. En Krishnamurthi, S. (ed.), Advances in agricultural sciences and their applications, pp. 317-331. Agricultural CollegeResearch Institute, Coimbatore.

HURRELL, J. A. \& G. DELUCCHI. 2014. Aportes de la Etnobotánica al estudio de las invasiones biológicas. Casos en la región rioplatense (Argentina). Historia Natural (3ra. Serie) 3 (2) (en prensa).

HURRELL, J. A., E. ULIBARRI, G. DELUCCHI \& M. L. POCHETTINO. 2010. Frutas frescas, secas y preservadas. En Hurrell, J. A. (ed.), Biota Rioplatense XV. Edit. Lola, Buenos Aires. 304 pp.

HURRELl, J. A., G. DELUCCHI, H. A. KELlER, P. C. STAMPELLA \& E. L. GUERRERO. 2012. Bryophyllum (Crassulaceae): especies ornamentales naturalizadas en la Argentina. Bonplandia 21: 7385.

IBODA. 2014. Instituto de Botánica Darwinion. Base de Datos. Flora del Cono Sur. Disponible: <http:// www2.darwin.edu.ar/Proyectos/FloraArgentina/ FA.asp $>$ [Consulta: I-2014].

JANICK, J. 2005. The origin of fruits, fruit growing, and fruit breeding. En J. Janick (ed.), Plant Breeding Review 25, pp. 255-320. Wiley, Hoboken.

KELLER, H. A., P. C. STAMPELLA, G. DELUCCHI \& J. A. HURRELL. 2013. Vernicia fordii y Aleurites moluccanus (Euphorbiaceae) en la Argentina. Naturalización y Etnobotánica. Bol. Soc. Argent. Bot. 48: 553-561.

KIM, M. J., H. J. PARK, M. S. HONG, H. PARK, M. S. KIM, K. H. LEEM, J. B. KIM, Y. J. KIM \& H. K. KIM. 2005. Citrus reticulata Blanco induces apoptosis in human gastric cancer cells SNU 668. Nutr. \& Cancer 51: 78-82.

KOEHLER-SANTOS, P., A. L. DORNELLES \& L. B. FREITAS. 2003. Characterization of mandarin citrus germplasm from southern Brazil by morphological and molecular analyses. Pesq. Agropec. Brasil. 38: 797-806.

KUJAWSKA, M., F. ZAMUDIO \& N. I. HILGERT. 2012. Honey-based mixtures used in home Medicine by nonindigenous population of Misiones, Argentina. Evid.-Based Complement. Altern. Med. 2012, doi:10.1155/2012/579350.

KUMAR, S., K. N. NAIR \& S. N. JENA. 2013. Molecular differentiation in Indian Citrus L. (Rutaceae) inferred from nrDNA ITS sequence analysis. Genet. Resour. Crop Evol. 60: 59-75.

KYNDT, T., T. N. DUNG, P. GOETGHEBEUR, H. T. TOAN \& G. GHEYSEN. 2010. Analysis of ITS of the rDNA to infer phylogenetic relationships among Vietnamese Citrus accessions. Genet. Resour. Crop Evol. 57: 183-192.

LAGUNA LUMBRERAS, E. \& G. MATEO SANZ. 2001.
Observaciones sobre la flora alóctona valenciana. Fl. Montiberica 18: 40-44.

LURO, F. F. LAIGRET, J. M. BOVÉ \& P. OLLITRAULT. 1995. DNA Amplified Fingerprinting, A Useful Tool for Determination of Genetic Origin and Diversity Analysis in Citrus. Hort. Sci. 30:1063-1067.

MABBERLEY, D. J. 1997. A classification for edible Citrus. Telopea 7: 167-172.

. 1998. Australian Citreae with notes on other Aurantioideae (Rutaceae). Telopea 7: 333-344.

2001. Citrus reunited. Australian Plants 21(166): $52-54$.

- 2004. Citrus (Rutaceae): A review of recent advances in Etymology, Systematics and Medical applications. Blumea 49: 481-498.

MACHADO, M. A., H. D. COLlETA FILHO, M. L. TARGON \& J. POMPER. 1996. Genetic relationship of Mediterranean mandarins (Citrus deliciosa Ten.) using RAPD markers. Euphytica 92: 321-326.

MARLYKYNTI, H., K. SURENDRA \& R. SATYAWADA. 2011. Karyological studies in ten species of Citrus (Rutaceae) of North-East India. Comp. Cytogen. 5: 277-287.

MARTÍNEZ-CROVETTO, R. 1963. Esquema fitogeográfico de la provincia de Misiones (República Argentina). Bonplandia 1: 171-223.

MARTIUS, C. F .P. 1843. Systema Materiae Medicae Vegetabilis Brasiliensis: Citrus, pp. 29-30. Fleischer, Lipsiae.

MIRANDA, D. E., A. V. BOHREN, H. KELLER, L. A. GRANCE \& H. M. GARTLAND. 2000. Clave de reconocimiento de especies leñosas de Rutaceae presentes en la Selva Paranaense (Argentina), mediante el uso de caracteres dendrológicos. Quebracho 8: 47-55.

MOREIRA NOVELLI, V., M. A. MACHADO \& C. ROMERO LOPES. 2000. Isoenzymatic polymorphism in Citrus spp. and Poncirus trifoliata (L.) Raf. (Rutaceae). Gen. Mol. Biol. 23: 163-168.

NEW SOUTH WALES FLORA ON LINE. 2013. Disponible: <http://plantnet.rbgsyd.nsw.gov.au> [Consulta: XI-2014].

NICOLOSI, E. 2007. Origin and taxonomy. En Khan, I. A. (ed.), Citrus Genetics, Breeding, and Biotechnology, pp. 19-43. CABI International, Wallingford.

, Z. N. DENG, A. GENTILE, S. LA MALFA, G. CONTINELLA \& E. TRIBULATO. 2000. Citrus phylogeny and genetic origin of important species as investigated by molecular markers. Theor. Appl. Genet. 100: 1155-1166.

PALACIOS, J. 1978. Citricultura Moderna. Hemisferio Sur. Buenos Aires. 409 pp.

PATIÑO, V. 1963. Plantas cultivadas y animales domésticos en la América equinoccial. IV. Plantas introducidas. Imprenta Departamental, Cali. 673 pp.

PEDERSEN, T. M. 1995. Especies de plantas vasculares introducidas y naturalizadas en el Noroeste 
Correntino. Anales Acad. Nac. Agron. Vet. 49: 1117.

PENJOR, T., M. YAMAMOTO, M. UEHARA, M. IDE, N. MATSUMOTO, R. MATSUMOTO \& Y. NAGANO. 2013. Phylogenetic relationships of Citrus and its relatives based on matK gene sequences. Plos One 8(4): e62574, doi: 10.1371/ journal.pone.0062574.

PIRANI, J. R. 2014. Citrus. En Lista de Espécies da Flora do Brasil. Jardim Botânico do Rio de Janeiro. Disponible: <http://floradobrasil.jbrj.gov. br $>$ [Consulta: II-2014].

PLANTS PROFILE USDA. 2013. Disponible: < http:// plants.usda.gov/> [Consulta: XI-2014].

PYŠEK, P. \& D. M. RICHARDSON. 2006. The biogeography of naturalization in alien plants. J. Biogeography 12: 2040-2050.

——, - M. REJMÁNEK, G. WEBSTER, M. WILLIAMSON \& J. KIRSCHNER. 2004. Alien plants in checklists and floras: towards better communication between taxonomists and ecologists. Taxon 53: 131-143.

QUINLAN, M. 2005. Considerations for collecting freelists in the field: examples from Ethnobotany. Field Methods 17: 1-16.

RAMÓN-LACA, L. 2003. The introduction of cultivated citrus to Europe via northern Africa and the Iberian Peninsula. Econ. Bot. 57: 502-514.

RAMOS-HURTADO, A. M., O. C. KOLLER, J. ARAÚJO MARIATH, I. A. SARTORI, S. THEISEN \& B. REIS. 2006. Diferenciação floral, alternância de produção e uso de ácido giberélico em tangerineira 〈Montenegrina〉 (Citrus deliciosa Ten.). Rev. Bras. Frutic. 28: 355-359.

RANDALL, R. 2012. A Global Compendium of Weeds. 2nd ed. Dept. Agric. Food, Perth. 1119 pp.

RICHARDSON, D. M., P. PYŠEK, M. REJMÁNEK, M. G. BARBOUR, F. DANE PANETTA \& C. J. WEST. 2000. Naturalization and invasion of alien plants: concepts and definitions. Diversity Distrib. 6: 93-107.

RICHARDSON, D. M., PYŠEK, P. Y CARLTON, J. T. 2011. A compendium of essential concepts and terminology in invasion ecology. En Richardson, D. M. (ed.), Fifty Years of Invasion Ecology: The Legacy of Charles Elton, pp. 409-420. WileyBlackwell, Oxford.

RIVERA NÚÑEZ, D., C. OBÓN, S. RÍOS, C. S. FERRÁNDEZ, F. MENDEZ, A. VERDE \& F. CANO. 1998. Las variedades tradicionales de frutales de la Cuenca del Rio Segura. Catálogo Etnobotánico 1, pp. 187-189. Serv. Publ. Univ. Murcia.

ROOSE, M. L., R. K. SOOST \& J. W. CAMERON. 1995. Citrus (Rutaceae). En Smartt, J. \& N. W. Simmonds (eds.), Evolution of crop plants. 2nd ed., pp. 443449. Wiley-Blackwell, Oxford.

SAINT HILAIRE, A. 1825. Flora Brasiliae Meridionalis. I: 338-339. A. Belin, Paris.

SAMAAN, L. G. 1982. Studies on the origin of Clementine tangerine (Citrus reticulata Blanco). Euphytica 31: 167-173.

SÁNCHEZ DAMAS, J. J., E. AVITIA GARCÍA, A. M. CASTILlO GONZÁlEZ, A. VILLEGAS MONTER \& T. CORONA TORRES. 2006. Estudio anatómico de la poliembrionía en tres portainjertos de cítricos. Revista Chapingo, Ser. Hort. 12: $145-$ 152.

SCORA, R. 1975. On the history and origin of Citrus. Bull. Torrey Bot. Club 102: 369-375.

SENASA. 2012. El cultivo del mandarino (Citrus reticulata) en Argentina. Servicio Nacional de Sanidad y Calidad Alimentaria, Buenos Aires. 12 pp.

SEO, M. N. \& C. XIFREDA. 2008. Rutaceae. En Anton, A. M. \& F. O. Zuloaga (eds.), Flora Fanerogámica Argentina 106: 1-22.

SIB. 2014. Sistema de Información de Biodiversidad. Administración de Parques Nacionales. Disponible: <http://www.sib.gov.ar/ficha/ PLANTAE*citrus*reticulata $>$ [Consulta: II-2014].

SPIEGEL-ROY, P. \& E. E. GOLDSCHMIDT. 1996. The Biology of Citrus. Cambridge University Press, Cambridge. 230 pp.

STAMPELLA, P. C.; G. DELUCCHI y M. L. POCHETTINO. 2013. Naturalización e identidad del "limón mandarina", Citrus $\times$ taitensis (Rutaceae, Aurantioideae) en la Argentina. Bol. Soc. Argent. Bot. 48: 161-169.

STEPP, J. R. 2005. Advances in ethnobiological field methods. Field Methods 17(3): 211-218.

SUlTANA, H. S., M. ALÍ \& B. P. PANDA. 2012. Influence of volatile constituents of fruit peels of Citrus reticulata Blanco on clinically isolated pathogenic microorganisms under in-vitro. Asian Pacif. J. Trop. Biomed. 2012: S1299-S1302.

SWINGLE, W. T. 1943. The botany of citrus and its wild relatives of the orange subfamily En Webber, H. J. \& L. D. Batchelor (eds.), The citrus industry I, 1st ed., pp. 129-474. University of California Press, Berkeley.

\& P. C. REECE. 1967. The botany of citrus and its wild relatives. En Reuther, W., H. J. Webber \& L. D. Batchelor (eds.), The citrus industry I, 2nd ed., pp. 190-430. University of California Press, Berkeley.

TANAKA, T. 1954. Species problem in citrus (Revisio aurantiacearum IX). Japanese Society for the Promotion of Science, Tokio. $152 \mathrm{pp}$.

. 1966. Hodgson's Citrus classification discussed. Bull. Univ. Osaka Prefecture, Ser. B, 18: 25-29.

1969. Misunderstanding with regard to Citrus classification and nomenclature. Bull. Univ. Osaka Prefecture, Ser. B, 21: 139-141. 
1977. Fundamental discussion of Citrus classification. Studia Citrologica 14: 1-6.

THE PLANT LIST. 2013. The Plant List. Version 1.1. Disponible: <http://www.theplantlist.org> [Consulta:XI-2014].

TROPICOS.ORG. 2014. Missouri Botanical Garden. Disponible: <http://www.tropicos.org $>$ [Consulta: XI-2014].

UZUN, A. \& T. YESILOGLU. 2012. Genetic Diversity in Citrus. En Çalişkan, M. (ed.), Genetic Diversity in Plants, pp. 213-230. InTech, Rijeka.

WEBBER, H. 1943. Cultivated varieties of Citrus. En Webber, H. \& L. Batchelor (eds.), The Citrus Industry. History, Botany, and Breeding, pp. 475668. University of California Press, Berkeley.
XIANG, C. \& M. L. ROOSE. 1988. Frequency and characteristics of nucellar and zygotic seedlings in 12 citrus rootstocks. Scientia Hort. 37: 47-59.

ZAMUDIO, F. \& N. I. HILGERT. 2011. Mieles y plantas en la medicina criolla del norte de Misiones, Argentina. Bonplandia 20:165-184.

ZHANG, D. \& D. J. MABBERLEY. 2008. Citrus. En Wu, Z. Y., P. H. Raven \& D. Y. Hong, (eds.), Fl. of China 11: 90-96. Science Press, Beijing - Missouri Botanical Garden Press, St. Louis.

ZHANG, Y., Y. SUN, W. XI, Y. SHEN, L. QIAO, L. ZHONG, X. YE \& Z. ZHOU. 2014. Phenolic compositions and antioxidant capacities of Chinese wild mandarin (Citrus reticulata Blanco) fruits. Food Chem. 145: 674-680.

Original recibido el 1 de junio de 2014; aceptado el 8 de noviembre de 2014. 